\title{
N-terminal residues regulate proteasomal degradation of AANAT
}

\begin{abstract}
Serotonin $N$-acetyltransferase (AANAT) catalyzes the conversion of serotonin to $N$-acetylserotonin, which is the immediate precursor for formation of melatonin. Although it is known that AANAT is degraded via the proteasomal proteolysis, detailed mechanisms are not defined. In this paper, we tested the in vivo role of proteasome inhibition on AANAT activity and melatonin release and examined the amino acid residues in AANAT that contribute to the proteasome degradation. We have shown that inhibition of proteasome activities in vivo in the intact pineal gland fails to prevent the light-induced suppression of melatonin secretion. Furthermore, in cell lines stably expressing AANAT, inhibition of proteasomal proteolysis, which resulted in a large accumulation of AANAT protein, similarly failed to increase AANAT enzyme activity proportional to the amount of proteins accumulated. Site-directed mutagenesis analysis of AANAT revealed that the AANAT degradation is independent of lysine and the two surface cysteine residues. Deletion analysis of $\mathrm{N}$-terminus identified the second amino acid leucine (L2) as the key residue that contributes to the proteasomal proteolysis of AANAT protein. These results suggest that rat AANAT protein is degraded via the $\mathrm{N}$-end rule pathway of proteasomal proteolysis and the leucine at the $\mathrm{N}$-terminus appears to be the key residue recognized by $\mathrm{N}$-end rule pathway.
\end{abstract}

\section{Zheping Huang*, Tiecheng Liu and Jimo Borjigin}

Department of Molecular and Integrative Physiology, University of Michigan Medical School, Ann Arbor, MI, USA

\section{Introduction}

Melatonin is a neurohormone synthesized and released from the pineal gland at night in all vertebrate animals tested to date. The nocturnal synthesis of melatonin is activated by dramatic increases in pineal serotonin $\mathrm{N}$-acetyltransferase (or arylalkylamine $\mathrm{N}$-aceyltransferase; AANAT) activity, which is stimulated by the adrenergic signaling from the superior cervical ganglia and controlled by the central circadian pacemaker in the brain [1]. When the adrenergic signaling is terminated by light at night, melatonin release declines rapidly with associated decrease in AANAT protein content [2, 3]. How AANAT is degraded is not fully understood.

Earlier studies demonstrate that the threonine residue at the 29th position (T29; T31 in sheep) is phosphorylated in vivo in the night at pineal gland, and the phosphorylation is associated with increased AANAT protein content [4]. Upon phosphorylation at the T29 residue, AANAT interacts with 14-3-3 proteins, which leads to the stabilization of AANAT protein in vitro [5]. Accordingly, mutations that affect either AANAT phosphorylation at the T29 position or a subsequent interaction with 14-3-3 would affect AANAT protein stability in vivo. Recent studies from our laboratory demonstrate that the H28Y mutation affects the ability of the phosphorylated AANAT-H28Y mutant to interact with 14-3-3 [6]. Additionally, studies have shown that AANAT isolated from the night pineal gland is tightly associated with 14-3-3 and is phosphorylated at the T31 position (equivalent to the T29 residue in rat) in sheep AANAT [5]. These studies demonstrate that AANAT phosphorylation and its subsequent interaction with 14-33 is a key event in stabilization of AANAT protein in vivo.

While phosphorylation and subsequent interaction with 14-3-3 increase AANAT protein levels, proteasomal activities dramatically reduce AANAT protein. When pineal cells are stimulated in vitro with a combination of betaadrenergic activator and inhibitor, AANAT activity and protein levels are low compared with those stimulated with the activators alone [2]. When proteasome inhibitor is added, the drug prevents the decrease in AANAT protein and activity (when assayed in broken cell extracts) elicited by the beta-adrenergic inhibitor [2]. In flow through cultures of rat retinae, light-induced suppression of melatonin release and AANAT activity is prevented by coincubation with a proteasome inhibitor [7]. These studies demonstrate that proteasome proteolysis of AANAT is one mechanism responsible for light-induced suppression of AANAT protein (and activity) and melatonin formation. The amino acid residues in AANAT that are targeted by the proteasome machinery are not defined.

Ubiquitin-mediated proteasomal degradation comprises the major proteolytic pathway in eukaryotes. Proteins targeted for degradation by this pathway are conjugated by ubiquitin chains to lysines via a complex cascade [8]. In addition to the canonical lysine ubiquitination, cysteines can also be targeted for ubiquitination in a few reported cases $[9,10]$. Moreover, the $\mathrm{N}$-terminus of a protein can 
also be tagged by an ubiquitin chain [9]. Studies have shown that when the amino acids targeted by the ubiquitin chains are removed or mutated to other residues, the resultant proteins can be markedly stabilized and no longer subjected to the proteasome degradations. Furthermore, many proteins with short lives are degraded via the $\mathrm{N}$-end role pathway [10], which relates the in vivo half-life of a protein to the identity of its $\mathrm{N}$-terminal residue. The destabilizing residues at the $\mathrm{N}$-terminus of short-lived proteins include leucine, phenylalanine, tyrosine, etc., whose substitutions to serine, alanine or other smaller residues stabilize the protein [11].

To define residues important for proteasomal degradation of AANAT, we have expressed rat AANAT proteins in HEK 293 cells from both wildtype and mutant AANAT constructs and analyzed their protein content with or without the proteasome inhibitor, MG132. Our results strongly suggest that the $\mathrm{N}$-terminus of AANAT mediates the proteasomal degradation of AANAT protein.

\section{Materials and methods}

\section{Animals}

All animal protocols were reviewed and approved by the University of Michigan animal care and use committee. Adult inbred PVG rats (Harlan, Indianapolis, IN, USA) were used in this study. Animals were housed in temperature-controlled chambers with a light and dark cycle of 12:12 hr (lights on at $6 \mathrm{hr}$ ).

\section{In vivo measurement of melatonin release using pineal microdialysis}

Rats were implanted with pineal microdialysis probes [12]. Following a recovery period of 1 day, animals were placed in microdialysis chambers. Microdialysis was performed with artificial CSF solution flowing continuously at $2 \mu \mathrm{L} /$ min. The pineal dialysates were collected in a sample loop of an automated injector (Instech, Plymouth Meeting, PA, USA), which delivers samples into an HPLC column (Sigma, St Louis, MO, USA) at $10 \mathrm{~min}$ intervals with the aid of an HPLC pump (Shimadzu, Tokyo, Japan). Fractionated pineal dialysates were analyzed online by a fluorescence detector. The automated control of the HPLC system including the handling and storage of the chromatograms were carried out with an external computer using Shimadzu chromatography software [12].

A light pulse of $10 \mathrm{~min}$ was given to rats on the third day of online microdialysis $4 \mathrm{hr}$ into the darkness (22:00$22: 10 \mathrm{hr}$ ) in the presence or absence of drug infusion. Isoproterenol (ISO, $1 \mu \mathrm{M}), \mathrm{MG} 132(1$ or $10 \mu \mathrm{M}$ ), or vehicle (DMSO) was individually infused into the pineal gland of rats for $4 \mathrm{hr}$ (20:00-2:00 hr) prior to the light pulse.

\section{Cell culture, mutagenesis, transfection, and treatment}

The detailed method on the generation of AANAT expressing plasmid can be found elsewhere [13]. Constructs with lysine mutations all contain a C-terminal myc epitope [14]. Point mutation was made using a QuikChange Site-
Directed Mutagenesis Kit (Stratagene, La Jolla, CA, USA). N-terminal truncation of AANAT was made using PCR method. All mutant constructs were confirmed by sequencing before transfection. HEK 293 cells were seeded in 24well plates at $70 \%$ confluency. Plasmid constructs were transfected the next day using Lipofectamine 2000 (Invitrogen, Carlsbad, CA, USA) according to the manufacture's recommendation. One day after transient transfection, or $24 \mathrm{hr}$ following splitting of the stably transfected cells, carbobenzoxy-L-leucyl-L-leucinal (MG132; Sigma), or vehicle (DMSO) was added to the media for indicated amount of time.

\section{Western blot analysis}

The pineal glands were collected from rats left in the darkness or exposed to light for $10 \mathrm{~min}(22: 00-22: 10 \mathrm{hr})$ and sacrificed at 23:00 hr in the dark. Total proteins from either the pineal glands, or AANAT transfected (transient or stable) cells were extracted and separated by electrophoresis and transferred to a nitrocellulose membrane. The membrane was blocked with 5\% nonfat dry milk in TBS-T solution (20 mM Tris- $\mathrm{HCl}, \mathrm{pH} 7.5,140 \mathrm{~mm} \mathrm{NaCl}$, and $0.05 \%$ Tween-20), and hybridized with anti-AANAT (generated in our lab, see [13]), anti-myc (generous gift from Dr. Jeremy Nathans), and anti-14-3-3 (Cat\# K-19 from Santa Cruz, Santa Cruz, CA, USA) antibodies. The secondary antibodies used include: IRDye 700- and IRDye 800conjugated anti-rabbit or anti-mouse IgG antibodies (LICOR Biosciences, Lincoln, NE, USA). The infrared signals were detected by the Odyssey Image System and analyzed by the Odyssey software (LI-COR Biosciences, Lincoln, NE, USA).

\section{Generation of HEK 293 cell lines stably expressing rAANAT}

HEK 293 stable cell lines were made essentially as described [3]. HEK 293 cells were grown in DMEM containing 10\% fetal bovine serum and $1 \%$ penicillin/streptomycin in a humidified $5 \% \mathrm{CO}_{2}$ incubator at $37^{\circ} \mathrm{C}$. Cells at $80-90 \%$ confluency were transfected with AANAT plasmids (rat AANAT wt coding fragments cloned in pCS2 vector; $0.9 \mathrm{mg}$ per well of a 24 -well plate) mixed individually with a puromycin resistance plasmid $(0.1 \mathrm{mg})$ using Lipofectamine 2000 (Invitrogen, Carlsbad, CA, USA). The transfected cells were split $24 \mathrm{hr}$ later at a 1:10 dilution into two $10-\mathrm{cm}$ plates. Puromycin $(2 \mathrm{mg} / \mathrm{mL})$ was added to the media the following day and incubation continued for 14 additional days until colonies were detected with naked eyes. Cells in each clone were transferred to a well in a 24-well plate using cloning cylinders and trypsin. Two to three days later, AANAT expression level of these closes was examined using Western blot. The clones that had highest level of expression were selected for experiments in this paper.

\section{AANAT activity measurement in intact cells}

Cells stably expressing AANAT, 2 days after reseeding, were incubated with either MG132 $(1 \mu \mathrm{M})$ or vehicle (DMSO) overnight. 5-methoxytryptamine (5-MT, $1 \mu \mathrm{M})$ 
was then added to the tissue culture cells for $4 \mathrm{hr}$, which is converted to melatonin (released into the tissue culture supernatant) by AANAT in the stable cells. Tissue culture supernatant was collected for melatonin measurement using HPLC coupled with a fluorescence detector.

\section{Results}

In order to obtain a precise timing of melatonin and AANAT protein suppression by light at night, a group $(n=5)$ of inbred PVG rats were analyzed using online microdialysis for multiple days. Prior to the light pulse, melatonin levels increased to the maximum levels at 21:40 hr, stayed at the peak levels for $7 \mathrm{hr}$, and began to decline at 5:00 hr (Fig. 1A). A light pulse given at 22:00 hr (white dashed line), resulted in an immediate suppression of melatonin secretion, which reached its minimum levels within $20 \mathrm{~min}$ and stayed at that levels for about $2 \mathrm{hr}$. Melatonin secretion was recovered to its nocturnal high levels $4 \mathrm{hr}$ following the end of the light pulse (at 22:10 hr). In all tested rats, melatonin secretion was the lowest during 22:30-23:30 hr following a 10-min light pulse at 22:00 $\mathrm{hr}$ (Fig. 1A).

AANAT protein levels in the same strain of rats showed dramatic decrease following the light pulse of $10 \mathrm{~min}$ from rats sacrificed at 23:00 hr (Fig. 1B). This level represents the minimum amount of AANAT protein contents following the light pulse in this strain of rats.

Since the nightly production of melatonin is the result of adrenergic stimulation of the pineal gland, we tested the ability of an exogenously infused adrenergic agonist, isoproterenol (ISO), to antagonize the action of light on melatonin release in vivo. When the pineal gland was directly infused with the ISO $(1 \mu \mathrm{M})$ prior to the light pulse,

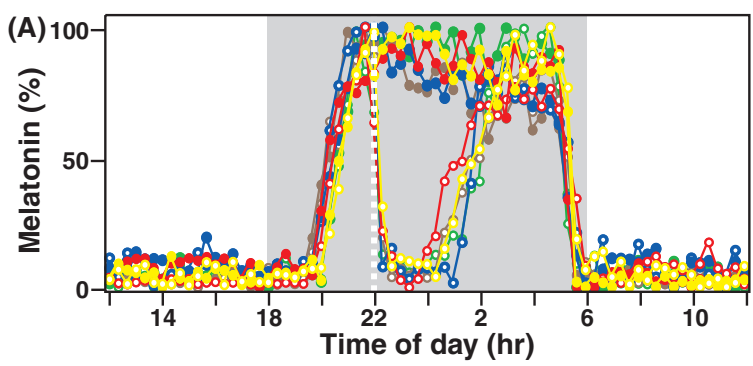

(B)
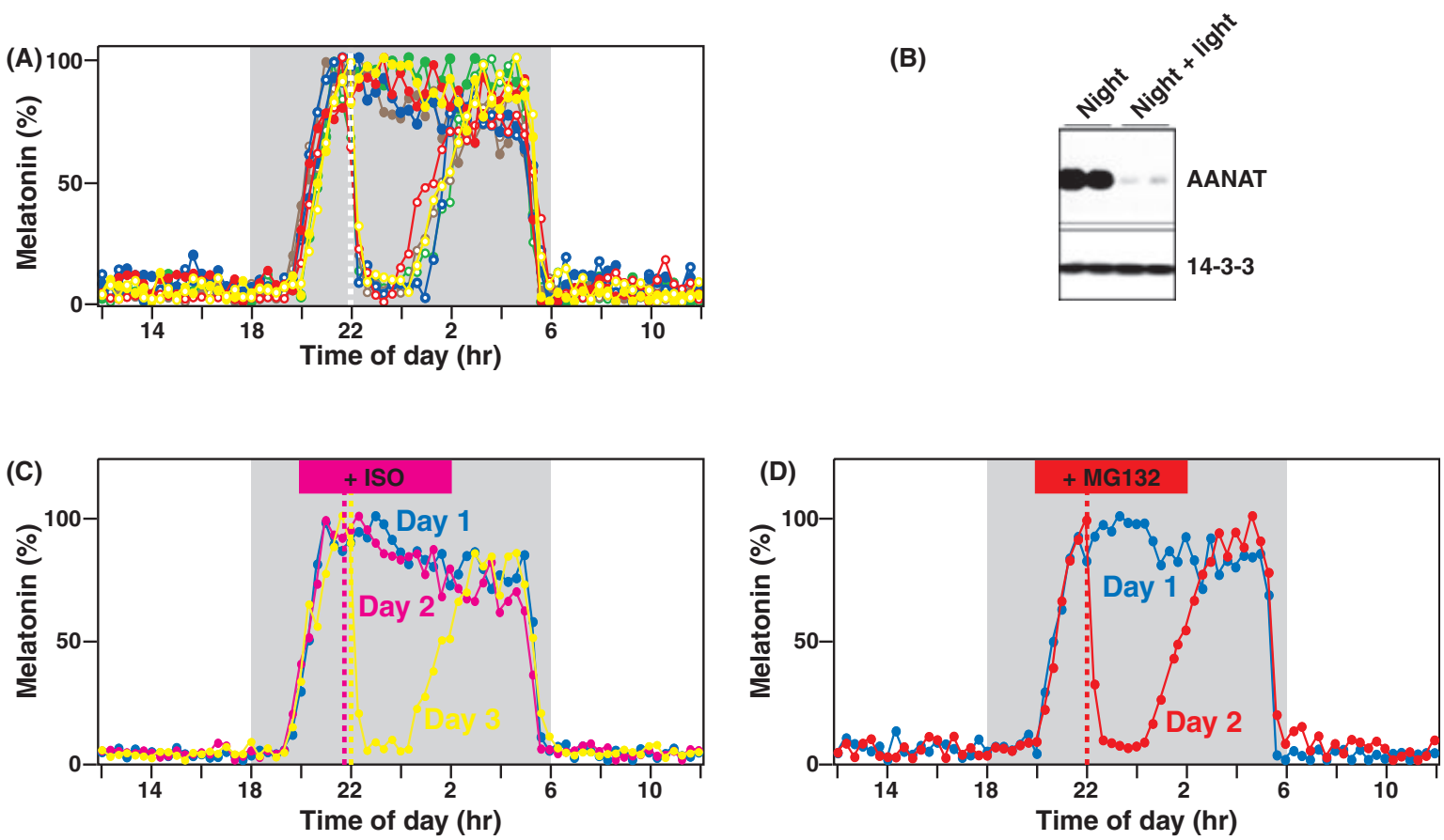

Fig. 1. Effects of light pulses at night on melatonin secretion and serotonin N-acetyltransferase (AANAT) protein levels in vivo. Melatonin secretion during each cycle was normalized to the maximum value of each night. Each data point represents the amount of melatonin in pineal dialysates during a 10-min collection ( $20 \mu \mathrm{L}$ of dialysates). The shaded gray areas represent the dark period (18:00-6:00 hr). (A) In vivo circadian profile of melatonin secretion from five rats without (solid dots) and with (white filled dots) light pulses (22:00-22:10 hr). Each color represents one rat. The 10-min light pulse, marked by a white dashed line, was given on day 3 of the microdialysis. (B) The effect of a single light pulse $(10 \mathrm{~min})$ at night on AANAT protein levels in rats. Two rats housed in LD 12:12 hr were exposed to a single light pulse for $10 \mathrm{~min}(22: 00-22: 10 \mathrm{hr}$, lights off at 18:00 hr), while two control rats were kept in the darkness without light exposure. All rats were sacrificed at 23:00 hr in the dark. Pineal protein extracts from rats without the light pulse (left two lanes) or with the light pulse (right two lanes) were loaded in each lane (one-fifth of a single pineal for each lane) and analyzed for expression levels of AANAT (top panel) and 14-3-3 (bottom panel) proteins. AANAT protein content was dramatically reduced following the light pulse. (C) Isoproterenol (ISO) given prior to light exposure is sufficient to prevent light-induced suppression of melatonin secretion in vivo. Melatonin secretion from a single rat was followed for three consecutive days. Blue tracings indicate the melatonin secretion patterns under normal conditions (LD 12:12 hr) on day 1. On day 2 (pink tracings), $1 \mu \mathrm{M}$ of ISO was infused into the rat pineal gland for $6 \mathrm{hr}(20: 00-2: 00 \mathrm{hr})$ and a 10-min light pulse was given at 22:00 hr (marked by a pink dashed line). Light-induced suppression of melatonin was prevented by ISO. On day 3 (yellow tracings), a light pulse was given (time marked as yellow dashed line) without the infusion of ISO, and there was a dramatic suppression of melatonin secretion similar as shown in panel A. These results were confirmed in more than five rats. (D) MG132 did not prevent the light-induced suppression of melatonin secretion. On day 1 (blue tracings), no infusion and no light pulse at night were given. On day 2 (red tracings), MG132 (1 $\mu \mathrm{M})$, from 20:00-2:00 hr was infused into the rat pineal. A light pulse was given at 22:00-22:10 hr (red dashed line), and there was a dramatic suppression of melatonin secretion. Identical results were obtained in at least three rats with this dose and in four rats with $10 \mu \mathrm{M}$ MG132 (data not shown). 
light-induced suppression of melatonin was completed prevented (Fig. 1C).

Inhibition of proteasome activity was shown previously to block the effect of light on AANAT degradation in pineal organ culture [2] and to prevent light-induced melatonin suppression in retina cultures [7]. We therefore tested the ability of a proteasome inhibitor in prevention of light-induced suppression of melatonin secretion in freely moving rats. In contrast to ISO (Fig. 1C), MG132 (1 and $10 \mu \mathrm{M})$, a potent cell permeable inhibitor of proteasome, was unable to prevent the light-induced suppression of melatonin secretion in vivo (Fig. 1D).

To further understand mechanisms of AANAT protein regulation, we generated cell lines stably expressing rat AANAT protein and stimulated cells with various drugs. When cells were exposed to either phorbal 12-myristate 13acetate (PMA), which stimulates protein kinase C (PKC), or forskolin (FSK), which elevates the intracellular levels of cAMP by activation of adenylyl cyclase, AANAT protein levels were clearly elevated (Fig. 2A). When AANATexpressing cells were exposed to MG132, AANAT protein levels were dramatically elevated in a duration dependent manner (Fig. 2B), confirming that the proteasome activity regulates AANAT protein degradation.

To understand why MG132 failed to prevent the lightinduced suppression of melatonin secretion in vivo, we tested AANAT activity in intact cells stably expressing AANAT in the presence of MG132. MG132 incubation of the AANAT-expressing cells resulted in an abundant accumulation of AANAT protein, with high molecular weight bands that might represent ubiquitinated proteins (Fig. 3A). When MG132-treated live cells were tested for AANAT activity, however, there was only a moderate increase in AANAT enzyme activity compared to the control cells without the inhibitor (Fig. 3B). When the AANAT activity was normalized by the amount of protein elevated by the proteasome inhibition, the AANAT enzyme

(A)

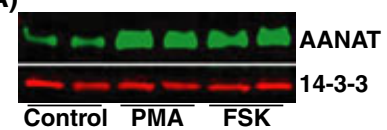

(B)

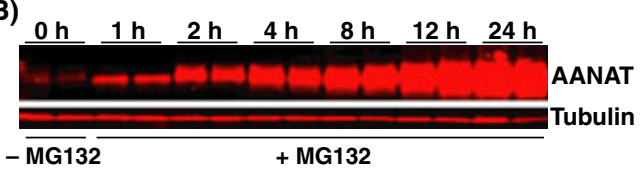

Fig. 2. Serotonin $N$-acetyltransferase (AANAT) protein content can be increased via kinase activation or proteasome inhibition in vitro. (A) PKA and PKC activators (PMA and FSK) increased the content of the AANAT protein. HEK 293 cells stably expressing AANAT were split into a 24-well plate, and stimulated with PMA $(1 \mu \mathrm{M})$, FSK $(10 \mu \mathrm{M})$ or DMSO (control) for $4 \mathrm{hr}$, then subjected to Western blot analysis for AANAT (upper panel), or 14-3-3 (low panel) protein content. (B) AANAT levels increase dramatically upon MG132 administration. HEK 293 cells stably expressing AANAT were stimulated with MG132 $(1 \mu \mathrm{M})$ for different periods of time and then subjected to Western blot for analysis of AANAT protein level. AANAT protein levels increased with stimulation by MG132. Each experiment was repeated in triplicate. activity was markedly reduced ( $8 \%$ of the control levels) per unit of expressed protein (Fig. 3C).

To define the amino acid residues in AANAT that are important for proteasome proteolysis of AANAT, we performed a series of site-directed mutagenesis. Four lysine residues are found in rat AANAT and are relatively conserved in mammalian AANAT protein sequences [15]. Each lysine was mutated to arginine singly or in combinations. When the lysine mutants were tested in HEK 293 cells in a Western analysis, no detectable changes of their protein levels were found for any of the mutant constructs, compared with the wildtype (Fig. 4A). Wildtype (wt), K8R (lysine at amino acid position \#8 was mutated to arginine), and lysine-less (K0) mutants (in which all four lysines were replaced with arginine), all of which were tagged with myc epitope [14] at the C-terminus, were transiently expressed in HEK 293 cells and were exposed to MG132 for $24 \mathrm{hr}$. Lysine-less mutant (K0) as well as K8R mutant displayed MG132 dependent elevation of protein contents to a similar extent as wildtype AANAT protein (Fig. 4B).

Reports indicate that, in some protein, the ubiquitination can occur on the cysteine residues [9]. Of the 10 cysteine residues found in rat AANAT, 6 cysteines (C37, C61, C75, $\mathrm{C} 158, \mathrm{C} 177$, and C191) were conserved across all mammalian species examined [15]. Of these, two (C37 and C61) were found on the surface of the crystal structure [16]. When they were mutated to alanine, their expression levels (Fig. 5A) as well as their sensitivity to MG132 (Fig. 5B) were unchanged from the wildtype AANAT.

A series of truncation mutations were generated that deleted 30 (not shown), 10, 5, 4 (not shown), 3 (not shown), and 2 amino acids from the N-terminus of AANAT, and were expressed individually in HEK 293 cells (Fig. 6). All $\mathrm{N}$-terminal truncation mutants displayed higher levels of mutant proteins compared with wildtype. When cells expressing these mutants were exposed to MG132, no further protection was evident compared to the wildtype AANAT protein.

The common feature among all truncation mutants was the absence of the leucine residue at the second position (L2). In fact, the amino acid residues from all other mammalian AANAT proteins at the same position are all bulky residues (proline, tryptophan, or asparagines, in addition to leucine). Unlike rat AANAT, which is composed of 205 amino acids, most other mammalian AANAT proteins are composed of 207 amino acids with additional dipeptides such as MS (methionine and serine) or MP (methionine and proline) added to their N-terminus [15]. Moreover, 9 out of the 11 AANAT proteins with the length of 207 amino acids contain serine at its second position (S2). To clarify the role of S2, a smaller amino acid compared with L2, in AANAT stability, we added MS to the N-terminus of rat AANAT and tested their sensitivity to MG132. Addition of a smaller amino acid ahead of the L2 did not alter the sensitivity of the mutant protein to MG132 compared to the wildtype AANAT (Fig. 6).

\section{Discussion}

AANAT is a key enzyme in synthesis of melatonin, a hormone with many physiological roles [17-21]. Melatonin 

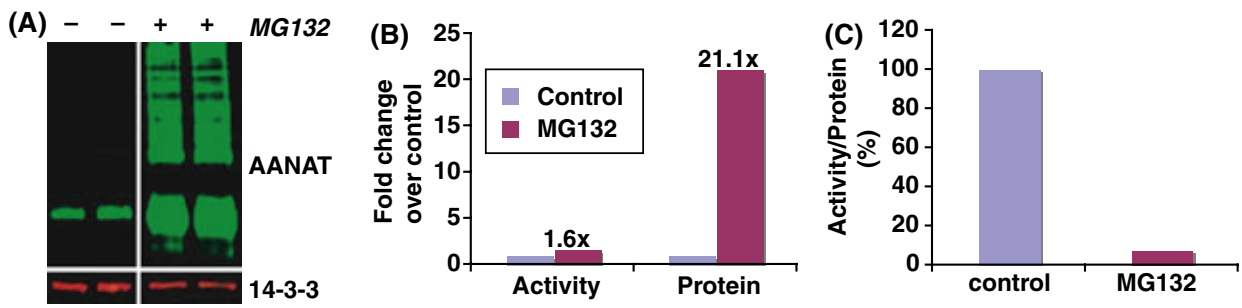

Fig. 3. The proteasome inhibitor MG132 increased serotonin N-acetyltransferase (AANAT) protein content without associated increase in enzyme activity. (A) HEK 293 cells stably expressing AANAT were stimulated with MG132 for $24 \mathrm{hr}$, and then subjected to Western blot using AANAT antibody (cells incubated with vehicle DMSO served as control). The AANAT protein content increased dramatically, with smearing of the upper bands. (B) The increase in AANAT protein content did not accompany corresponding increase in AANAT enzyme activity. AANAT protein content following MG132 incubation increased by 21 -fold compared with the control (right columns). AANAT activity assayed in intact cells increased by 1.6-fold. (C) The enzyme activity of AANAT (normalized by the amount of protein) is much lower in cells stimulated by MG132 (compared to the untreated control).
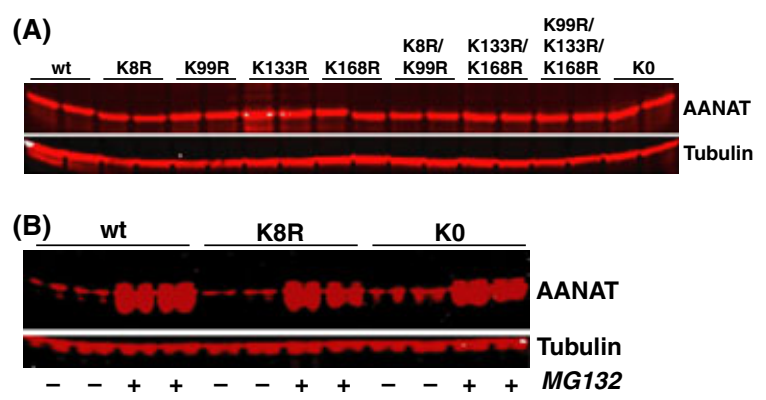

Fig. 4. Lysine residues do not contribute to the proteasomal degradation of serotonin $\mathrm{N}$-acetyltransferase (AANAT). (A) Lysine mutants are expressed at similar levels as AANAT wildtype. Each of the four lysine residues found in rat AANAT were mutated to arginine singly or in combinations. All lysine mutants, as well as the wt AANAT constructs, containing a C-terminal myc tag, were transfected into HEK 293 cells, and subjected to Western blot analysis after $48 \mathrm{hr}$ to determine the AANAT protein levels. There was no detectable change in the AANAT protein level for any of the lysine mutants. (B) The sensitivity of the lysine-less AANAT protein to MG132 is similar to that of wildtype AANAT. Wildtype (wt), K8R (lysine at amino acid position \#8 was mutated to arginine), and a lysine-less (K0) mutant (all four lysines were replaced with arginine) were transiently transfected into HEK 293 cells and were exposed to $10 \mu \mathrm{M}$ MG132 or DMSO for $24 \mathrm{hr}$. The elevation of protein content after exposure to MG132 in K8R and $\mathrm{K} 0 \mathrm{mu}-$ tants is similar to the wildtype AANAT protein. Each experiment was repeated in triplicate.

synthesis and AANAT activity are well known to be sensitive to light at night, due to the rapid degradation of AANAT protein by the proteasome [2]. As the proteasome proteolysis begins with ubiquitination on a specific amino acid residue on a target protein, it is important to clarify the identity of the amino acid residues involved in AANAT degradation. In this paper, we have performed a series of mutagenesis analysis of rat AANAT and identified the Nterminus as the critical region controlling the proteasomal degradation of AANAT.

While many studies have examined the kinetics of melatonin suppression by light, very few studies followed melatonin secretion in the same animal before and after the light pulse at high resolution and correlated that with AANAT protein levels. Our studies, using the pineal
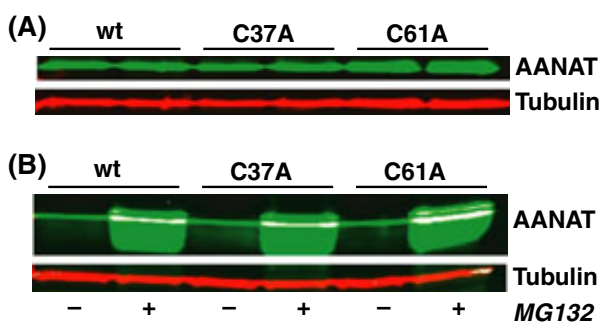

Fig. 5. Mutation of surface cysteine residues does not affect the stability of serotonin $\mathrm{N}$-acetyltransferase (AANAT) or its response to MG132. (A) Mutation of the two cysteines (at amino acid positions \#37 and 61), found on the surface of the AANAT crystal structure, did not alter the mutant protein levels. The cysteine residues were singly mutated to alanine (C37A and C61A) and were transiently transfected into HEK 293 cells and subjected to Western blot analysis. The protein levels of these cysteine mutants were similar to the control. (B) The sensitivity of the cysteine mutants to MG132 is similar to the wildtype AANAT. Each experiment was repeated in triplicate.

microdialysis technique [12], demonstrate that following a light pulse of $10 \mathrm{~min}$, melatonin secretion was suppressed to less than $50 \%$ of maximum levels within 20 min and stayed at minimum levels for more than $80 \mathrm{~min}$ in all animals. Importantly, our study was performed in freely behaving inbred rats with minimum inter-individual differences in their melatonin secretion profiles [22] and consequently showed little variation in their response to light. These results increased our confidence in using individual rats in analysis of AANAT protein expression, which showed dramatic suppression following a 10-min light pulse. Importantly, even when melatonin secretion was at its minimum following the light pulse, AANAT protein was still found at detectable levels when compared to its daytime levels. At early night, this amount of AANAT protein was sufficient to ensure near maximum levels of melatonin production in our previous studies [23]. These results suggest that AANAT degradation alone may not account fully for the precipitous decline of melatonin secretion by light.

Light pulses at night suppress melatonin secretion by terminating the release of norepinephrine from the superior cervical ganglion. It is thus expected that agonists of 


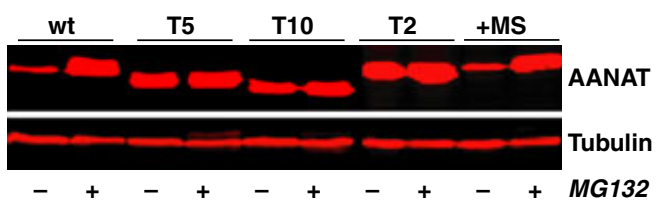

Fig. 6. The N-terminal residues of AANAT are important for the stability of the protein. A series of N-terminal truncation mutants were made by deletion of the first 30, 20, 10 (T10), 5 (T5), 4, 3, and 2 (T2) amino acids. The truncated mutants with an engineered methionine residue at its $\mathrm{N}$-terminus were transiently transfected into HEK 293 cells and exposed to $10 \mu \mathrm{M}$ of MG132 for $24 \mathrm{hr}$ (no exposure served as control). Cells were collected and subjected to Western blot analysis for AANAT protein content. The protein content of all truncated mutants was much higher than that of the wildtype AANAT, and treatment with MG132 made little difference in the protein content of the mutants. When methionine and serine residues were added to the $\mathrm{N}$-terminus of wildtype rat AANAT (+MS), the expression levels of the mutant protein was comparable to the wildtype rat AANAT. Furthermore, the MSmutant responded to MG132 in a similar manner as wildtype AANAT protein. This experiment was repeated in triplicate.

adrenergic receptors are capable of blocking the effect of light at night. Our studies performed in the same animal before and after the light pulse in the presence of drugs demonstrate that this is indeed the case: direct infusion of an adrenergic agonist, isoproterenol, into the pineal gland of a living rat before the light pulse was able to prevent the suppression of melatonin secretion. Since the rapid AANAT degradation parallels the precipitous decline of melatonin secretion and is due to proteasomal proteolysis, we anticipated that melatonin suppression by light could be prevented in vivo by blocking the proteasome activity. To our surprise, MG132 was unable to block the light-induced suppression of melatonin secretion, despite the use of multiple different doses that were all effective in blocking AANAT protein degradation in tissue culture cells. Our data contradict with those performed in cultured retinae, which showed that suppression of proteasome activity could restore melatonin secretion in retina cultures [7].

To understand degradation mechanisms of AANAT, we generated stable cell lines expressing rat AANAT in HEK 293 cells. Drugs that activate the signaling pathways (PKC by PMA and PKA by FSK), which are stimulated in vivo by norepinephrine release at night, markedly elevated AANAT protein expression in these cells. Moreover, inhibition of the proteasome activity by MG132 resulted in a dramatic increase of AANAT protein levels in a time dependent manner. When live cells with accumulated AANAT protein were assayed for AANAT enzyme activity, however, the increase in activity was barely significant. In contrast with the 21-fold increase in AANAT protein content in the presence of MG132, a merely 1.6-fold increase of activity was detected. Thus, the accumulated AANAT protein expressed only $8 \%$ of the enzyme activity per unit of protein compared with controls that were treated with the vehicle (DMSO). These results from the live tissue culture cells support our in vivo findings described above, which shows that inhibition of proteasome activity was unable to suppress light-induced inhibition of melatonin secretion. It remains to be determined whether similar results can be obtained with other cell permeable proteasome inhibitors besides MG132.

Since lysine is the canonical ubiquitination site on target protein for the proteasomal degradation [8], we tested each of the four lysines present in rat AANAT. Mutation of lysine to arginine singly, in pairs, or altogether caused no change in the stability of the mutant AANAT protein. Furthermore, the sensitivity of the lysine-less AANAT mutant protein to the inhibitor of the proteasome was unchanged compared to the wildtype AANAT. These results demonstrate strongly that AANAT degradation by the proteasome is independent of the lysine residues.

In addition to the canonical lysine ubiquitination, proteasome degradation pathway can also target cysteine residues $[24,25]$. To examine the role of cysteine residues in AANAT degradation, we mutated two of the conserved cysteines found on the surface of the protein structure [16] that are most likely to be accessed by the proteasome machinery. Mutations of either one of the cysteines resulted in no change in protein levels when expressed in HEK 293 cells, and no alteration was found in their sensitivity to proteasome inhibitors compared with wildtype AANAT protein. These results suggest that the two surface cysteine residues, $\mathrm{C} 37$ and $\mathrm{C} 61$, do not contribute to the proteasomal proteolysis of AANAT.

In addition to the pathways described above, proteasomal proteolysis is also achieved via the N-end-rule pathway [11], which is an ubiquitin-dependent proteolytic system where ubiquitin ligases (N-recognins) bind to type 1 and type 2 destabilizing $\mathrm{N}$-terminal residues of protein substrates [26]. The type 1 residues include basic amino acids such as lysine, arginine, etc., whereas the type 2 residues include bulky hydrophobic amino acids such as leucine, tryptophan, and tyrosine [26]. On the other hand, smaller residues such as serine or alanine are considered to be the stabilizing amino acids at corresponding positions. To test the role of the $\mathrm{N}$-terminus in AANAT degradation, we performed a series of deletion analysis from its $\mathrm{N}$-terminus that removed the first 30, 20, 10, 5, 4, 3, and 2 amino acids. All truncated AANAT proteins were markedly stabilized when expressed in HEK 293 cells and became insensitive to proteasome inhibition. The common feature among all deletion mutations was the absence of the leucine residue at the second position from the N-terminus, which is a bulky hydrophobic amino acid considered to be one of the type 2 destabilizing residues in the $\mathrm{N}$-end role pathway [26]. These results strongly suggest that AANAT degradation follows the N-end rule pathway of proteasomal proteolysis and the leucine 2 is important in this process.

As serine is considered to be the stabilizing N-terminal residue, and is found at the $\mathrm{N}$-terminus of many mammalian AANAT proteins including human, sheep and degu [15], we added Met-Ser (MS) dipeptide to the N-terminus of rat AANAT. The resultant mutant protein, 207 amino acids in length, contained MSML in its N-terminus, which is similar to that of Monkey and Degu AANAT proteins that contain MSMQ at the N-terminal ends [15]. The mutant protein, despite possessing a stabilizing serine at position 2, behaved like rat AANAT in its low expression and responsiveness to proteasome inhibition when expressed in HEK 293 cells. These studies suggest that: (i) 
serine at position \#2 may not always be the stabilizing residue in the $\mathrm{N}$-end rule pathway or (ii) the key destabilizing residue in the $\mathrm{N}$-end rule pathway can be close to, but not necessarily at the very $\mathrm{N}$-terminus of a short lived protein. It remains to be determined how AANAT from other species is degraded with serine in their N-termini. Furthermore, the appropriate context surrounding the destabilizing N-terminal resides in AANAT remains to be explored in future studies.

\section{Acknowledgements}

We thank Mr. Dan Allen for help with animal care, and Ms. Yaxi Chen for laboratory assistance. This study was partially supported by NIH grant NS057583 (to JB) and DoD grant FA9550-08-0149 (to JB).

\section{References}

1. Chattoraj A, Liu T, Zhang LS et al. Melatonin formation in mammals: In vivo perspectives. Rev Endocr Metab Disord 2009; 10:237-243.

2. Gastel JA, Roseboom PH, Rinaldi PA et al. Melatonin production: proteasomal proteolysis in serotonin N-acetyltransferase regulation. Science 1998; 279:1358-1360.

3. Huang Z, Liu T, Chattoraj A et al. Posttranslational regulation of TPH1 is responsible for the nightly surge of 5-HT output in the rat pineal gland. J Pineal Res 2008; 45:506-514.

4. Choi BH, Chae HD, Park TJ et al. Protein kinase C regulates the activity and stability of serotonin $\mathrm{N}$-acetyltransferase. J Neurochem 2004; 90:442-454.

5. Ganguly S, Gastel JA, Weller JL et al. Role of a pineal cAMP-operated arylalkylamine N-acetyltransferase/14-3-3binding switch in melatonin synthesis. Proc Natl Acad Sci USA 2001; 98:8083-8088.

6. Huang Z, Chattoraj A, Li X et al. The increased degradation of NAT-H28Y mutant protein is due to a reduced interaction with 14-3-3. J Pineal Res 2009; 46:119-120.

7. Fukuhara C, Dirden JC, Tosini G. Photic regulation of melatonin in rat retina and the role of proteasomal proteolysis. Neuroreport 2001; 12:3833-3837.

8. Roos-Mattuus P, Sistonen L. The ubiquitin-proteasome pathway. Ann Med 2004; 36:285-295.

9. CAdwell K, Coscoy L. Ubiquitination on nonlysine residues by a viral E3 ubiquitin ligase. Science 2005; 309:127-130.

10. Ravid T, Hochstrasser M. Diversity of degradation signals in the ubiquitin-proteasome system. Nat Rev Mol Cell Biol 2008; 9:679-690.
11. Varshavsky A. The N-end rule: functions, mysteries, uses. Proc Natl Acad Sci USA 1996; 93:12142-12149.

12. BorJigin J, LiU T. Application of long-term microdialysis in circadian rhythm research. Pharmacol Biochem Behav 2008; 90:148-155.

13. Huang Z, Deng J, Borjigin J. A novel H28Y mutation in LEC rats leads to decreased NAT protein stability in vivo and in vitro. J Pineal Res 2005; 39:84-90.

14. BorJigin J, NAthans J. Insertional mutagenesis as a probe of rhodopsin's topography, stability, and activity. J Biol Chem 1994; 269:14715-14722.

15. Lee SJ, Liu T, Chattoraj A et al. Posttranscriptional regulation of pineal melatonin synthesis in Octodon degus. J Pineal Res 2009; 47:75-81.

16. Hickman AB, Namboodiri MA, Klein DC et al. The structural basis of ordered substrate binding by serotonin $\mathrm{N}$-acetyltransferase: enzyme complex at $1.8 \mathrm{~A}$ resolution with a bisubstrate analog. Cell 1999; 97:361-369.

17. Borjigin J, Li X, SNyder SH. The pineal gland and melatonin: molecular and pharmacologic regulation. Annu Rev Pharmacol Toxicol 1999; 39:53-65.

18. Cervantes M, Morali G, Letechipia-Vallejo G. Melatonin and ischemia-reperfusion injury of the brain. J Pineal Res 2008; 45:1-7.

19. Fischer TW, Slominski A, Tobin DJ et al. Melatonin and the hair follicle. J Pineal Res 2008; 44:1-15.

20. Tengattini S, Reiter RJ, Tan DX et al. Cardiovascular diseases: protective effects of melatonin. J Pineal Res 2008; 44:16-25.

21. Reiter RJ, Paredes SD, Manchester LC et al. Reducing oxidative/nitrosative stress: a newly-discovered genre for melatonin. Crit Rev Biochem Mol Biol 2009; 44:175-200.

22. LiU T, BorJigin J. Relationship between nocturnal serotonin surge and melatonin onset in rodent pineal gland. J Circadian Rhythms 2006; 4:12.

23. LIU T, BorJIGIN J. N-acetyltransferase is not the rate-limiting enzyme of melatonin synthesis at night. J Pineal Res 2005; 39:91-96.

24. Ravid T, Hochstrasser M. Autoregulation of an E2 enzyme by ubiquitin-chain assembly on its catalytic residue. Nat Cell Biol 2007; 9:422-427.

25. Williams C, Van Den Berg M, Sprenger RR et al. A conserved cysteine is essential for Pex4p-dependent ubiquitination of the peroxisomal import receptor Pex $5 \mathrm{p}$. J Biol Chem 2007; 282:22534-22543.

26. TASAKI T, KWON YT. The mammalian N-end rule pathway: new insights into its components and physiological roles. Trends Biochem Sci 2007; 32:520-528. 